\title{
Modelo para la determinación del ratio de secado del matico y ortiga en la región sierra ecuatoriana
}

Model for the determination of the drying ratio of matico and nettle in the Ecuadorian mountain's region

1 Sebastian Alberto Guerrero Luzuriaga https://orcid.org/0000-0001-9512-2307

Docente - investigador Universidad Nacional de Chimborazo saguerrero@unach.edu.ec

2 Byron Adrián Herrera Chávez

https://orcid.org/0000-0003-1116-9939

Docente - investigador Universidad Nacional de Chimborazo

bherrera@unach.edu.ec

3 Ronald Mauricio Zurita Gallego iD https://orcid.org/0000-0001-8992-9008

Docente - investigador Escuela Superior Politécnica de Chimborazo

ronald.zurita@espoch.edu.ec

4 Dayana Maité Palacios Sotomayo (iD) https://orcid.org/0000-0002-8129-3479

Docente - investigador Universidad Nacional de Chimborazo

dayamaite195@gmail.com

Artículo de Investigación Científica y Tecnológica Enviado: 08/11/2021

Revisado: $23 / 11 / 2021$

Aceptado: $10 / 12 / 2021$

Publicado:05/01/2022

DOI: https://doi.org/10.33262/concienciadigital.v5i1.1963

\section{Cítese: Sotomayor, D. M. (2022). Modelo para la determinación del ratio de secado del matico y ortiga en la región sierra ecuatoriana. ConcienciaDigital, 5(1), 35-56. https://doi.org/10.33262/concienciadigital.v5i1.1963 \\ CONCIENCIA DIGITAL, es una Revista Multidisciplinar, Trimestral, que se publicará en soporte electrónico tiene como misión contribuir a la formación de profesionales competentes con visión humanística y crítica que sean capaces de exponer sus resultados investigativos y científicos en la misma medida que se promueva mediante su intervención cambios positivos en la sociedad. https://concienciadigital.org La revista es editada por la Editorial Ciencia Digital (Editorial de prestigio registrada en la Cámara Ecuatoriana de Libro con No de Afiliación 663) www.celibro.org.ec


Palabras claves:

Agroindustria,

Modelo

agroindustrial,

Metodología

proceso,

Competitividad.

\section{Keywords:}

Agroindustry, agro-industrial model, process methodology, competitiveness.

\section{Resumen}

Se precisa transferir ciencia y la tecnología en la mejora de las producciones agrícolas, a los productores de la región andina ecuatoriana,, y mejorar la comercialización de sus producciones. El objetivo de esta investigación es proveer modelo para la mejora de los productos del matico y ortiga en su procesamiento. Se realizaron secados experimentales de las plantas en un secador eléctrico tipo túnel de bandejas a temperaturas de $45 \mathrm{C}, 55 \mathrm{C}$ y 65 C con una duración de 6 horas para la temperatura de 45 C, 5 horas para $55 \mathrm{C}$ y 4 horas para $65 \mathrm{C}$, cada experimento con 4 repeticiones. Se adoptan estudios exploratorios, descriptivos, explicativos. Se apelan a métodos estadísticos-matemáticos, inductivos-deductivos, de expertos, análisis y síntesis de fuentes. Los resultados en la determinación de la ratio de secado de matico y ortiga se utilizó el modelo matemático de la curva característica basado en la ecuación y $=\mathrm{Ax}+\mathrm{B}$; obteniendo valores para la ortiga de $A=-0,0006 x 2-0,0704 x+1.9485$ y $B=-0,007 x-0,0136$; para el caso del matico $A=-0,0007 \times 2-0,0793 x+2,25$ y $B=-0,007 x-0,0184$ en todas las ecuaciones, $\mathrm{x}$, representa la temperatura de secado. Estas ecuaciones, obtuvieron valores de los cuales se formuló la ratio de secado con ecuación $\mathrm{RS}=\ln (\mathrm{mr})-\mathrm{A} / \mathrm{B}$, que se determina en un contenido de humedad del $12 \%$ al $14 \%$. Estos demuestran la viabilidad del modelo y su aplicación inmediata. Se concluye en socializar a productores de la provincia y la región sierra ecuatoriana este modelo de secado.

\section{Abstract}

It is necessary to transfer science and technology in the improvement of agricultural productions, to the producers of the Ecuadorian Andean region, and to improve the commercialization of their productions. The objective of this research is to provide a model for the improvement of matico and nettle products in their processing. Experimental drying of the plants was carried out in a tray tunnel type electric dryer at temperatures of $45 \mathrm{C}, 55 \mathrm{C}$, and $65 \mathrm{C}$ with a duration of 6 hours for a temperature of $45 \mathrm{C}, 5$ hours for $55 \mathrm{C}$, and 4 hours for $65 \mathrm{C}$, each experiment with 4 repetitions. Exploratory, descriptive, explanatory studies are adopted. They appeal to statistical-mathematical, inductive-deductive, expert methods, analysis, and synthesis of sources. The results in the determination of the drying ratio of matico and nettle was used the 
mathematical model of the characteristic curve based on the equation $\mathrm{y}=\mathrm{Ax}+\mathrm{B}$; obtaining values for nettle of $\mathrm{A}=-0.0006 \mathrm{x} 2-$ $0.0704 x+1.9485$ and $B=-0.007 x-0.0136$; for the case of the matico $\mathrm{A}=-0.0007 \mathrm{x} 2-0.0793 \mathrm{x}+2.25$ and $\mathrm{B}=-0.007 \mathrm{x}-0.0184$ in all equations, $x$, represents the drying temperature. These equations obtained values from which the drying ratio was formulated with the equation $\mathrm{RS}=\ln (\mathrm{mr})-\mathrm{A} / \mathrm{B}$, which is determined at a moisture content of $12 \%$ to $14 \%$. These demonstrate the viability of the model and its immediate application. It concludes in socializing producers of the province and the Ecuadorian highlands region this drying model.

\section{Introducción}

El Ecuador, cuenta con una gran diversidad de plantas medicinales y aromáticas como el matico, de la familia Piperaceae, Género Piper, nombre científico Piper aduncum "y que recibe los nombres comunes de Matico, hierba del soldado, achotlín o cordoncillo" (Varela, 2011). Sus flores, de la cuáles se extraen aceites esenciales (Shimabukuro y Torres, 1992).

Por otro lado, la ortiga, de la familia Urticaceae, Género Urtica, y de nombre científico Urtica dioica, recibe los nombres comunes de ortiga, la hierba de los ciegos, Urtica, según Quiroz (2013). Ambas plantas gozan de propiedades medicinales, reconstituyentes, terapéuticas y son una fuente de aceites esenciales útiles para el organismo. Comercializar estas plantas exige conocer de la ratio de secado, lo que hasta la fecha es un criterio empírico entre los productores y comercializadores, por lo que se precisa un procedimiento de cálculo de las ratios, capaz de aportar a la calidad del producto.

Relativo al estado del arte y mejores prácticas

Hoy en día, se disponen de varias opciones para el consumo de plantas medicinales al enfatizar en la calidad y prolongar la vida útil de estos productos. A partir de este enfoque, se ha planteado la presente investigación, al determinar el procedimiento, de la ratio de secado para el matico y ortiga, y en este caso de estudio, para los productores locales de la provincia de Chimborazo.

Así, se determinan las características físicas, se establece la varianza de humedad en función del tiempo y la temperatura. Estas variables permiten crear un modelo de ratio 
de secado ajustado a las variaciones de sus características. Se adopta en esta investigación, el enfoque de Torrecillas (2000) y Tabar (2011) en la interpretación del término de alimentos como la extracción deliberada del agua que contienen, que, en la mayoría de los casos, se ejecuta mediante la evaporación del agua por adición de su calor latente de evaporación.

Para la presente investigación, es definida la humedad relativa como la relación entre el peso del vapor de agua contenido en un kilogramo de aire saturado a una determinada temperatura. Significa la relación de presión de vapor de agua presente en el producto con respecto a la presión de saturación de vapor de agua. El contenido de humedad en equilibrio está definido por el tipo de producto de las plantas de matico y ortiga, por la temperatura y humedad.

La aplicación del secado, resulta operación clave y crítica en muchas industrias químicas, alimentarias y de transformación, al facilitar el manejo posterior del producto. Un correcto secado permite el empleo satisfactorio del producto y reduce costes de embarque y transporte; preserva los productos de fermentaciones durante el almacenamiento y el transporte; aumenta el valor o utilidad de los productos residuales.

La ratio de secado se define como el tiempo que transcurre desde que el secador es cargado con el producto húmedo, hasta que el producto haya alcanzado el contenido de humedad deseado. De los modelos matemáticos descritos en Tabar (2011), se adopta el estadístico inferencial al determinar las ecuaciones de regresión de las ratios de secado.

En el caso específico de las plantas aromáticas y medicinales, el secado para su conservación promueve el mantenimiento de los componentes del vegetal fresco, y de esta manera se evita la proliferación de microorganismos. Autores, al estilo de Contreras (2006), abundan en el uso de gases calientes para suministrar el calor en contacto directo con el alimento, fundamentalmente por convección, y arrastrar el líquido vaporizado y del secado indirecto.

Modelos y diseños de secadores, pueden ser estudiados según Jaramillo y Narváez (2012), como son de bandeja, de túnel, rotativos de cascada, de persianas periféricas, calentamiento indirecto y de lecho fluidizado.

Como bien afirman los autores Castellón y Espinoza (2009), es el calor la fuente de energía para la evaporación, donde su intensidad depende de la cantidad de energía suministrada por unidad de tiempo, además de la capacidad del medio donde se efectué el secado para absorber la humedad liberada por el producto.

La variación de humedad obtenida en el proceso de secado con respecto al tiempo, refiere el investigador Cabezas (2008), puede ser representada mediante curvas. El 
contenido de humedad de equilibrio está definido por el tipo de producto a ser secado, la temperatura y humedad, y el material higroscópico contenido de humedad.

Argumentan Cova y Marinelli (2011), referido a la variación de humedad, que es el valor de la humedad obtenido después de expuesto a un largo periodo de tiempo de secado, según las condiciones meteorológicas en las que se efectué el proceso.

Entonces, la ratio de secado en la presente investigación, es asumido como el tiempo que transcurre desde que el secador es cargado con el producto húmedo hasta que el producto haya alcanzado el contenido de humedad deseado. Como acertadamente enfatiza Rojas (2014), al someter un producto a un proceso de secado, se realizan pesadas a intervalos predefinidos y puede trazarse la curva del contenido en humedad frente al tiempo de secado

Este modelo de secado, expresado en calidad de procedimiento, aplica el principio de ratio de secado en matico y ortiga. De tal manera, es resuelto el problema en la asociación de agricultores de plantas aromáticas ubicada en la parroquia Llucut, ubicada en la región sierra en provincia Chimborazo. Así, se aporta el conocimiento del modelo de secado a la nueva empresa, dedicada al secado de plantas, y de tal manera, obtener mejores réditos económicos.

Este modelo de ratio, determina la dinámica del secado para aplicar en diferentes procesos a partir de simulación matemática en secadores artificiales, y permite el diseño eficiente de ratio de secado en el matico y ortiga. El modelo matemático de la curva característica es utilizado, a tenor de los presupuestos de Hernández (2011), para estandarizar las cinéticas de secado y modelarlas, de manera que puedan ser utilizadas a diferentes condiciones de humedad relativa, velocidad del aire y temperatura.

Problematización y preguntas científicas

La creación de pequeñas y medianas empresas Pymes en forma de asociación de agricultores de plantas aromáticas, goza de popularidad entre los habitantes de esta región sierra ecuatoriana. Tomando como campo de estudio la asociación localizada en la parroquia Llucut, región sierra en provincia Chimborazo, el modelo de secado responde a la necesidad de determinar las ratios, y de tal manera, obtener mejores réditos económicos.

Hasta la fecha, el proceso de secado y venta de estas plantas medicinales ha sido empírico, con diferentes niveles de aceptación y o rechazo en el mercado. Ello, provoca estancamiento de los inventarios en los puestos de venta, pérdidas de producciones, se encarecen los costos de procesamiento. De tal manera, el problema declarado, es el desconocimiento de la ratio óptima de secado de las plantas de matico y ortiga, a ser comercializados en los mercados serranos. 
Las preguntas por responder en esta investigación son, si es posible calcular la ratio óptima de secado que permita elevar la calidad de las plantas en el proceso de comercialización; si pueden adoptarse prácticas del estado del arte actual en función de perfeccionar el proceso de secado; de la capacidad de generalizar este modelo de secado al resto de Pymes encargadas de esta industria.

De las contradicciones dialécticas que generan esta investigación, la voluntad del estado ecuatoriano en mejorar la calidad de vida de las poblaciones indígenas en la región sierra, y la baja adopción de herramientas que permitan desarrollar sus procesos y ventas; del insuficiente nivel de vinculación a problemas sociales y la oportunidad de la Universidad nacional de Chimborazo, al soluciones necesidades de esta índole; de la existencia de asociaciones y Pymes y baja transferencia de conocimientos y herramientas para mejorar su desempeño social.

Objetivos y justificación de la investigación

El objetivo principal de la presente investigación, es proveer del modelo de secado expresado en procedimiento para determinar la ratio óptima de las plantas de matico y ortiga a ser comercializados en los mercados serranos.

De los objetivos específicos, son demostrar que es posible calcular la ratio óptima de secado para elevar la calidad de las plantas en el proceso de comercialización; adoptar prácticas del estado del arte actual en función de perfeccionar el proceso de secado; sugerir generalizar este modelo de secado al resto de Pymes encargadas de esta industria.

Se justifica esta investigación a partir de la conveniencia, expresada en el ámbito económico-social, al resolver necesidades de mejora de la calidad en el proceso de secado de plantas medicinales. Ello implica disminución de costos, mejor aceptación en el mercado y mayor competitividad. La relevancia social estriba en la mayor participación de asociados en el proceso de las plantas y ventas. Los actores beneficiados son las asociaciones y Pymes encargadas del proceso y ventas de plantas medicinales en la región sierra.

Las implicaciones prácticas de esta investigación residen en la solución de transferir el modelo y procedimiento de secado de las plantas a las asociaciones encargadas. Ello, resuelve problemas de diversas naturalezas al optimizar recursos laborales y humanos. Del valor teórico contenido en esta investigación se cita el propio modelo, revelado como herramienta al aportar el estado del arte en este campo. El aporte teórico radica en rellenar el hueco del conocimiento relativo a modelos de secado de plantas medicinales en asociaciones encargadas de esta tarea. 


\section{Metodología}

Esta investigación, declara su carácter científico, pues se define como la serie de pasos que conducen a la búsqueda de conocimientos mediante la aplicación de métodos y técnicas de corte científico. Así, se aplican técnicas e instrumentos estadísticosmatemáticos; modelación al algoritmo de acciones y tareas, además de procedimiento metodológico al optimizar y mejorar el procesamiento del matico y la ortiga en busca de mayor competitividad de los productores en la región sierra ecuatoriana.

\section{Tipos de estudios adoptados}

Se adoptan y declaran estudios de tipo exploratorio, pues el objetivo o tema a investigar es poco estudiado y no ha sido abordado con la profundidad requerida con anterioridad. Resultan insuficientes las fuentes referenciales en el Ecuador sobre este campo investigativo, de la ausencia de estudios y modelos desde la visión agroindustrial que permite optimizar y mejorar procesamientos de cultivos de matico y ortiga. Así, el estudio permite aumentar el grado de familiaridad con este problema poco estudiado en el contexto local, a partir de la propuesta del modelo en cuestión validado.

Se declara la investigación cuantitativa al medir y determinar los pesos y ratios de secado, de estudio de caso al ejecutar la investigación en Pyme serrana, de tipo explicativo al fundamentar los resultados e interpretar el impacto a la hora del proceso de secado.

La presente investigación, permite identificar conceptos y variables que permiten establecer prioridades para investigaciones posteriores o sugerir afirmaciones en cuanto a la adecuación de instrumentos para la determinación de tiempos de secado aplicando la ciencia y enfoques agroindustrial. La gestión informacional ejecutada, revela que existen nichos no investigados acerca de la disponibilidad de instrumentos metodológicos a generalizar entre las pequeñas y medianas empresas encargadas del procesamiento de estos cultivos.

Énfasis en esta investigación en el marco metodológico, que a tenor de Hurtado y Toro (2007), al afirmar que el diseño del marco metodológico constituye la medula de la investigación, se refiere al desarrollo propiamente del trabajo investigativo. El estudio de tipo descriptivo, se aplica a tenor de los postulados de Hernández et al. (2006), donde la investigación descriptiva busca especificar propiedades, características y rasgos importantes de cualquier fenómeno que se analice.

Así, se especifica el modelo de secado validado, revelado en fases al caracterizar los productos, el desarrollo estadístico inferencial, y determinación de los rangos óptimos de mejora. De tal manera, es posible evaluar dimensiones y componentes del problema científico. 
Referido al estudio explicativo, se logra desbordar la mera descripción de conceptos y fenómenos asociados al paradigma agrícola y complementado con campo industrial, estableciendo relaciones entre estos a partir del instrumento diseñado y validado, propuesto como herramienta de mejora y optimización. De tal manera, pueden responderse o explicarse enfoques y visiones desde disímiles campos de estudio, como es el económico, agroindustrial.

Se clasifica esta investigación como proyecto factible tipo propuesta. Se pretende solucionar problemas, requerimientos y necesidades a un problema específico, al diseñar, validar e implementar esta herramienta expresada como modelo metodológico a ser generalizado en las pequeñas y medianas empresas encargadas del procesamiento del matico y ortiga en la región sierra ecuatoriana.

Referido a la factibilidad de realización de esta investigación y asociado al proyecto factible, se adoptan los presupuestos de Arias (2006), el cual señala que se trata de una propuesta de acción para resolver un problema practico o satisfacer una necesidad. Es indispensable que dicha propuesta se acompañe de una investigación, que demuestre su factibilidad o posibilidad de realización.

El estudio de caso o de campo es declarado y ejecutado a tenor de los presupuestos de Roldan (2000), en calidad de análisis sistemático de problemas con el propósito de detallarlos, explicar sus causas y efectos, entender su naturaleza y los factores contribuyentes y predecir su ocurrencia en el propio medio donde se suscitan los eventos investigados. Así, este estudio parte del análisis a nivel de laboratorio, validado al comparar los niveles de mejora en los atributos físicos

Se revela el estudio de caso al diseñar, validar e implementar herramienta para determinar, mejorar y optimizar los tiempos de secado de productos agrícolas de matico y ortiga en la asociación de productores. Para tales fines es validado el modelo-procedimiento, ejecutando el diseño del experimento, evaluando resultados; discutidos los significados y mejoras a realizar como proyecto perfectible.

Métodos científicos declarados en la investigación

Asociado al conjunto de técnicas y procedimientos empleados para producir conocimiento, el método científico, se mencionan el método de análisis y síntesis bibliográfico de las fuentes estructuradas y no estructuradas, al conocer y contextualizar las mejores prácticas y estado del arte del campo de estudio y en específico del problema científico; método de consulta a expertos, al apelar a especialistas que evacuen las dudas y permitan reorientar el experimento, que provean del criterio de validez de la propuesta del conocimiento; método de las contradicciones dialécticas, lo que permite descubrir la 
génesis del problema científico y los elementos conflictuales que en ellos se revelan, desmembrando los efectos y razones.

Se adicionan el método del enfoque sistémico, permitiendo enlazar, relacionar de manera ínter e intra - disciplinar los procesos implícitos en este modelo, adoptando las relaciones y prácticas dependientes entre estos. Se declara el método estadístico-matemático, énfasis en la estadística inferencial y regresiones lineales.

De la operacionalización de las variables

Se adopta como variable independiente la ratio de secado de matico y ortiga, el cual resulta el período transcurrido desde que el secador es saturado con el producto en estado fresco hasta alcanzar el contenido de humedad esperado. Los indicadores por medir son la temperatura, tiempo, peso y las curvas de secado. Son establecidos los instrumentos y técnicas a aplicar (Guerrero, 2015).

La variable dependiente resulta la planta de matico y la planta herbácea ortiga, donde los indicadores a determinar es la humedad del producto en estado fresco y seco, se aplica la técnica de la determinación de la humedad.

Procesamiento y análisis

En esta investigación, el proceso de secado de matico y ortiga se realizó a temperaturas de $45^{\circ}, 55^{\circ}$ y $65^{\circ} \mathrm{C}$. esta decisión responde a que, en anteriores investigaciones, se ha determinado como temperaturas óptimas en el secado de plantas, al exceder estos rangos de temperatura de secado las plantas, pueden perder sus componentes volátiles.

Para efectuar el proceso de secado se desarrollaron las siguientes actividades de adquisición de materia prima, proporcionadas por diferentes huertas ubicadas en la parroquia San Luis perteneciente a la Provincia de Chimborazo. Se inspeccionó que las plantas estén recién cosechadas, libres de maleza, sin torceduras y en estado fresco con el fin de obtener datos precisos durante el proceso.

La preparación de las muestras, se realizó un cortado y un lavado. En el caso del matico se procedió a quitar las hojas del tallo las mismas que fueron sometidas al proceso de secado. La ortiga se cortó en ramas de $15 \mathrm{~cm}$ desde la flor. Una vez que las muestras han sido cortadas se procede a realizar el secado de estas. Previamente tomando muestras de 50 gramos y colocando papel aluminio en la superficie de las bandejas para evitar que se peguen.

Las muestras de matico y ortiga son colocadas en el secador a diferentes temperaturas. Por cada hora que dure el proceso de secado se debe registrar la perdida de humedad utilizando una balanza analítica; dejando enfriar el producto por un periodo de 10 minutos utilizando un desecador para evitar que las muestras absorban humedad. Para determinar 
el \% de humedad se utilizó una termo balanza cuyos datos fueron comprobados con un cálculo gravimétrico que relaciona la perdida de humedad y el porcentaje de masa seca.

En cuanto a la determinación de la variación de la humedad con respecto al tiempo, se desarrolló un control de pesos durante cada hora que se efectuó el proceso de secado.

\section{Resultados}

Estos resultados logrados en la presente investigación optimizan el proceso de secado de las plantas, mejoran la calidad y optimizan los tiempos y temperaturas de procesamiento. De tal manera, el modelo investigativo resulta:

\section{Figura 1}

Enfoque de la investigación

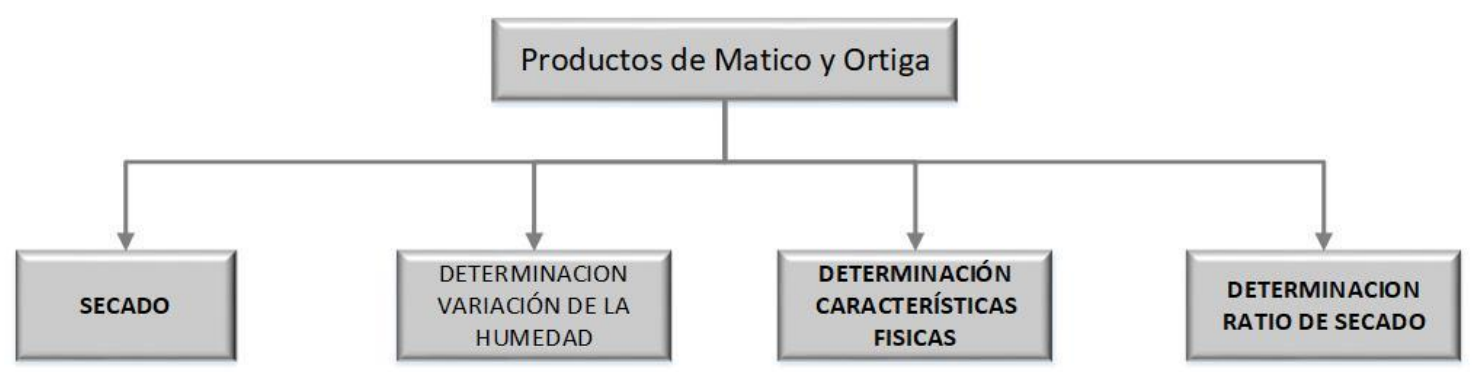

Así, la variable de secado, influye en las propiedades fisicoquímicas de las platas de matico y ortiga mejorando sus cualidades. Ejecutada la investigación, se evidencia la mayor aceptación por el mercado y el aumento de la competitividad de productores y vendedores. Se demuestra como la aplicación de la ciencia, expresada en el campo de la ingeniería agroindustrial, ayuda a resolver problemas y necesidades en la sociedad, y en este caso específico en las Pymes serranas.

Presentación del modelo

Esta investigación, aporta el modelo para el cálculo del secado óptimo de matita y ortiga, extrapolable en región sierra y otras regiones del Ecuador, asumiendo que las humedades, temperaturas ambientes difieren según sea región costa, oriente, insular, sierra. En la figura 2 se muestra el algoritmo del procedimiento 
Figura 2

Algoritmo del modelo

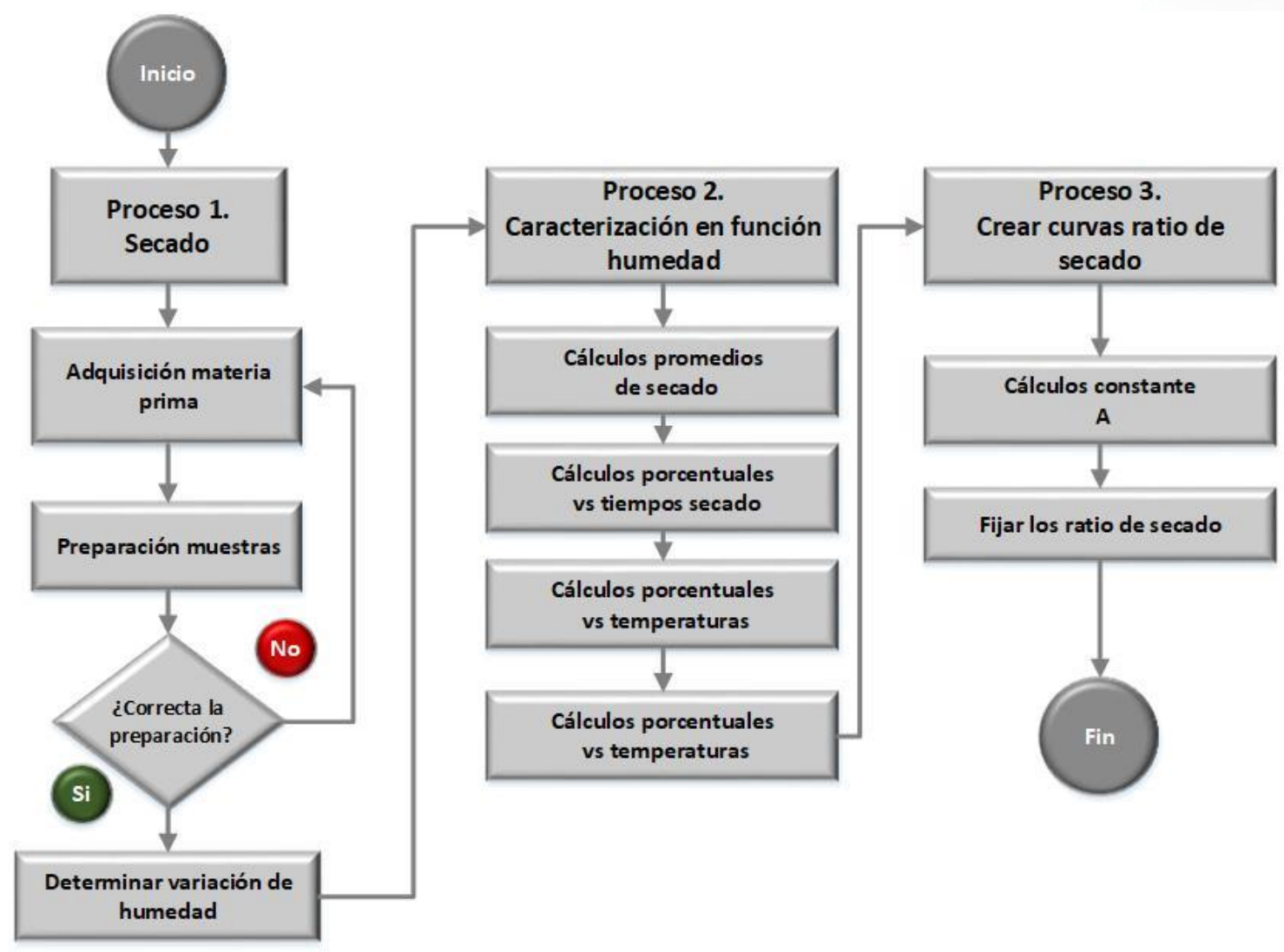

Este algoritmo enfatiza en la correcta selección y adquisición de la materia prima, así como la meticulosidad en la preparación de las muestras. Un error en estos pasos iniciales, impiden los cálculos correctos de la ratio de secado. Así, el proceso inicial resulta la clave del éxito para este procedimiento.

Los procesos dos y tres, caracterizados por la garantía de la infraestructura técnica de hornos y balanzas, cristalería y otros dispositivos de laboratorio. Ya los métodos de cálculo son descritos hasta fijar la ratio de secado.

En esta investigación, el proceso de secado de matico y ortiga se realiza a temperaturas de $45^{\circ}, 55^{\circ}$ y $65^{\circ} \mathrm{C}$. Tal decisión responde a que en otras investigaciones se ha determinado como temperaturas óptimas en el secado de plantas, pues al exceder estos rangos de temperatura de secado, las plantas pierden sus componentes volátiles.

Estos autores, enfatizan en el rigor profesional al muestrear, inspeccionar, cortar y pesar, como acciones que garanticen la objetividad del experimento. Se inspeccionó que las plantas estén recién cosechadas, libres de maleza, sin torceduras y en estado fresco con el fin de obtener datos precisos durante el proceso. 
Una vez que las muestras han sido cortadas, se procede a realizar el secado de estas. Son seleccionadas muestras de 50 gramos, colocadas en el secador a diferentes temperaturas. Por cada hora que dure el proceso de secado se debe registrar la perdida de humedad utilizando una balanza analítica dejando enfriar el producto por un periodo de 10 minutos utilizando un desecador para evitar que las muestras absorban humedad.

Para determinar la variación de humedad con respecto al tiempo, se desarrolló un control de pesos durante cada hora que se efectuó el proceso de secado. Para determinar el \% de humedad se utilizó una termo balanza cuyos datos fueron comprobados con un cálculo gravimétrico que relaciona la perdida de humedad y el porcentaje de masa seca.

De tal manera, se ilustra para el caso del matico el modelo de ratio de secado. Una vez deshidratado el matico, la planta presenta un color verde obscuro. Su olor se reduce con el proceso de secado y el sabor aumenta. Presenta una textura muy quebradiza, los talles y hojas son frágiles y se rompen fácilmente. El tamaño se reduce en un $40 \%$ debido a la eliminación de agua en el proceso de secado. La tabla 1, ilustra las características físicas del matico deshidratado en escala de 1 -10 según intensidad.

Tabla 1

Características físicas del matico deshidratado

\begin{tabular}{|c|c|c|c|c|c|c|c|c|c|c|}
\hline \multicolumn{2}{|c|}{ CARACTERÍSTICA } & 12 & 3 & 4 & 5 & 6 & 7 & 8 & 9 & 10 \\
\hline COLOR & Verde obscuro & & & & & & & $\mathrm{X}$ & & \\
\hline OLOR & Agradable & & & $\mathrm{X}$ & & & & & & \\
\hline SABOR & Aumenta & & & & & & $\mathrm{X}$ & & & \\
\hline TEXTURA & Quebradiza & & & & & & & & & $\mathrm{X}$ \\
\hline TAMAÑO & Reduce & & & & & & $X$ & & & \\
\hline
\end{tabular}

Se determinó la media de los pesos alcanzados en cada repetición realizada tomando en cuenta las temperaturas de secado. La humedad obtenida en el secado es el resultado del tiempo que transcurre durante el proceso. Es destacable que, en las primeras horas de secado la planta de matico elimina un gran porcentaje de agua. En esta investigación se determinó el porcentaje de humedad del matico en estado fresco que es de aproximadamente el $80 \%$.

Referido a la representación del porcentaje de humedad, la figura 3, muestra a 55 grados Celsius el valor 


\section{DDigital}

Figura 3

Representación porcentaje de humedad del matico

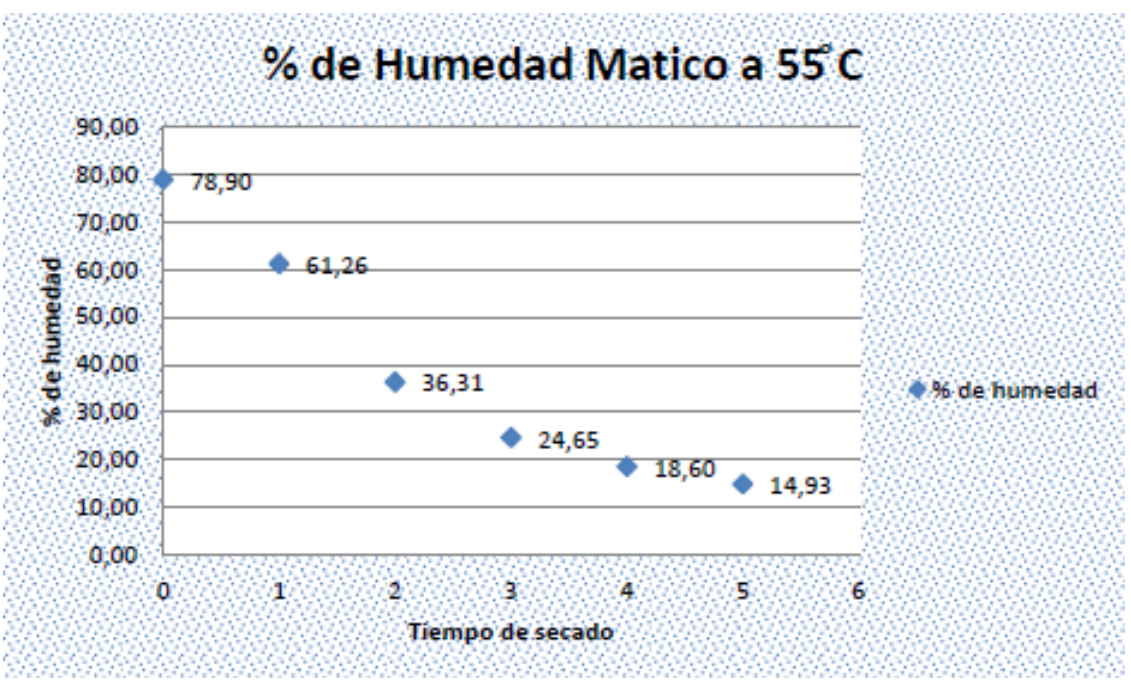

Al obtener la ecuación lineal de la ratio de secado a partir de los pesos promedios obtenidos de cada repetición, se determinó el logaritmo natural y la masa relativa con respecto al tiempo. Los datos obtenidos en la determinación del logaritmo natural y la masa relativa son representados mediante curvas cuyo valor de correlación es igual a 1 y se ejecutan para secado a 45, 55, 65 grados Celsius, como ilustran las tablas 2, 3, 4.

\section{Tabla 2}

Linealización de la ratio de secado del matico a $45^{\circ} \mathrm{c}$

\begin{tabular}{cccccccc}
\hline Tiempo & 0 & 1 & 2 & 3 & 4 & 5 & 6 \\
\hline $\begin{array}{c}\text { Masa } \\
\text { Relativa }\end{array}$ & 1,00 & 0,79 & 0,58 & 0,31 & 0,22 & 0,18 & 0,15 \\
\hline Ln (MR)* & 0,00 & $-0,24$ & $-0,55$ & $-1,18$ & $-1,52$ & $-1,69$ & $-1,91$ \\
\hline *Antilogaritmo masa relativa & & & & &
\end{tabular}

\section{Tabla 3}

Linealización de la ratio de secado del matico a $55^{\circ} \mathrm{C}$

\begin{tabular}{ccccccc}
\hline Tiempo & $\mathbf{0}$ & $\mathbf{1}$ & $\mathbf{2}$ & $\mathbf{3}$ & $\mathbf{4}$ & $\mathbf{5}$ \\
\hline $\begin{array}{c}\text { Masa } \\
\text { Relativa }\end{array}$ & 1,00 & 0,61 & 0,36 & 0,25 & 0,19 & 0,15 \\
\hline LN (MR)* & 0,00 & $-0,49$ & $-1,01$ & $-1,40$ & $-1,68$ & $-1,90$ \\
\hline${ }^{* \text { Antilogaritmo masa relativa }}$ & & & &
\end{tabular}




\section{Tabla 4}

Linealización de la ratio de secado del matico a $65^{\circ} \mathrm{C}$

\begin{tabular}{cccccc}
\hline Tiempo & $\mathbf{0}$ & $\mathbf{1}$ & $\mathbf{2}$ & $\mathbf{3}$ & $\mathbf{4}$ \\
\hline $\begin{array}{c}\text { Masa } \\
\text { Relativa }\end{array}$ & 1,00 & 0,52 & 0,29 & 0,19 & 0,15 \\
\hline Ln (MR)* & 0,00 & $-0,65$ & $-1,23$ & $-1,64$ & $-1,92$ \\
\hline *Antilogaritmo masa relativa & & &
\end{tabular}

Entonces, al realizar la linealización de la ratio de secado, se determinaron las curvas de secado del antilogaritmo con respecto al tiempo. Las ecuaciones lineales fueron las que más se ajustaron a un coeficiente de correlación de uno. Con la determinación de las curvas de secado, se aplica el modelo matemático de la curva característica obteniendo la ecuación $\mathrm{y}=\mathrm{Ax}+\mathrm{B}$, la misma que será utilizada en la determinación de las ratios óptimas en la cinética del secado del matico. Como ilustración, en la figura 4, se muestra la curva de Ln para la MR o masa relativa vs tiempo a $55^{\circ} \mathrm{C}$.

Figura 4

Curva LN MR vs tiempo a $55^{\circ}$ C para el matico

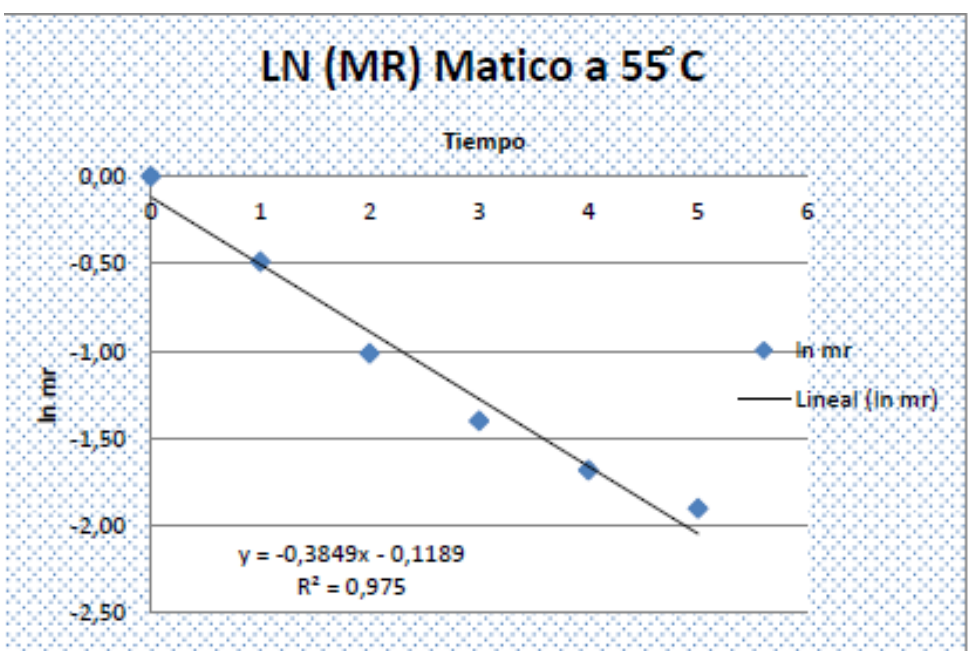

Con los resultados obtenidos durante el cálculo de linealización de la ratio del secado del matico y la representación mediante curvas de LN (MR) y MR con respecto al tiempo; se obtiene la constante de regresión representada con la letra "A". La constante "A" representa la eliminación de agua durante el proceso de secado según el tiempo que conlleva el proceso, tomando en cuenta distintas temperaturas de secado. De tal manera, en la figura 5 se pueden observar las curvas, variable A del matico. 


\section{Conciencia}

Figura 5

Variable A del matico

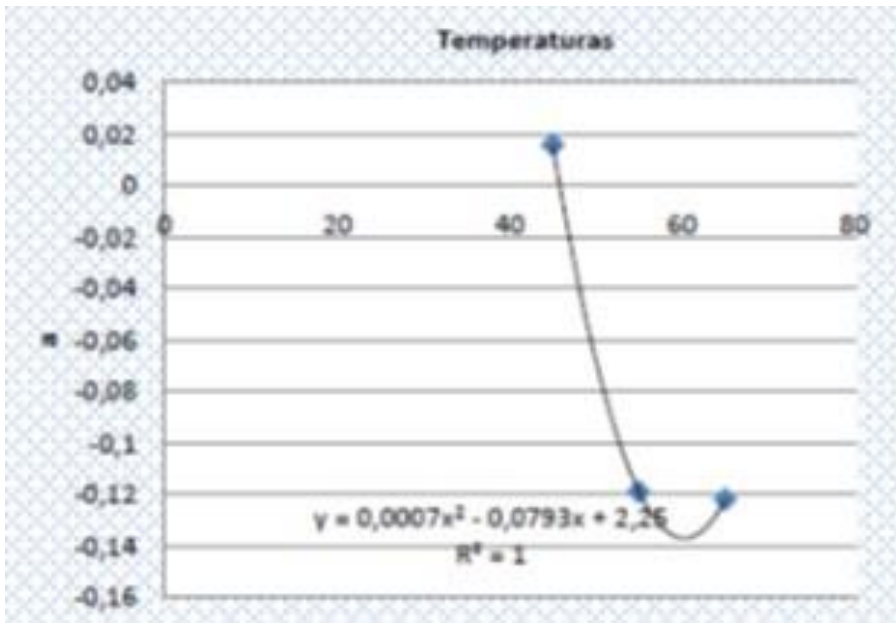

Según los datos logrados en la linealización de la ratio del secado del matico y la representación mediante curvas de LN (MR) y MR con respecto al tiempo, se obtiene la constante "B". Ésta constante determina la dinámica del secado del producto con relación a la temperatura a la que está expuesta, tomando en cuenta el \% de perdida de humedad durante el proceso. En la FIGURA 6, se puede observar la determinación de la constante "B" con respecto al tiempo.

Figura 6

Variable B del matico

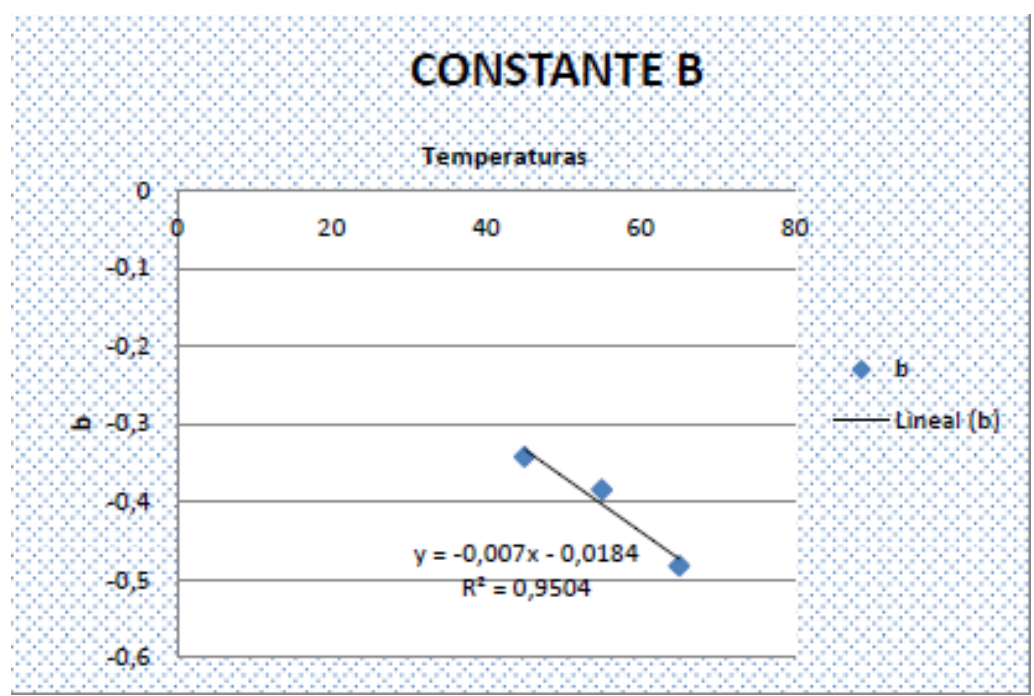


La ratio de secado es un parámetro muy importante que considerarse en la cinética del secado, constituye el tiempo que conlleva el proceso; generalmente este parámetro esta dado en horas o en días.

Para la determinación de la ratio de secado del matico se aplica las constantes de regresión obtenidas a partir de la linealización de la ratio. Con la determinación de la ratio de secado se pretende obtener una masa relativa de 0,15 gramos, debido a que varias investigaciones demuestran que al obtener este peso se ha eliminado un gran porcentaje de agua. De tal manera, en la tabla 5 se muestra la ratio de secado del matico a $55^{0} \mathrm{C}$.

Tabla 5

Ratio secado matico

\begin{tabular}{cc}
\hline TEMPERATURA & $\begin{array}{c}55 \text { grados } \\
\text { centígrados }\end{array}$ \\
\hline MASA & 0,15 gramos \\
RELATIVA & \\
\hline CONSTANTE “A” & 0,006 \\
\hline CONSTANTE “B” & $-0,4034$ \\
\hline RATIO DE & 5 horas \\
SECADO & \\
\hline
\end{tabular}

Para el caso de la determinación del modelo de secado de la ortiga, se procede de manera idéntica. Sus resultados de pesado y secado, con expuestos en Guerrero (2015). Así, para la determinación de la Constante A, la relación de temperatura respecto a los pesos gravimétricos es indicado en la tabla 6 .

\section{Tabla 6}

Determinación de constante a para la ecuación del modelo de secado

\begin{tabular}{cc}
\hline Temperaturas & Valores obtenidos \\
\hline $45 \mathrm{C}$ & $-0,0381$ \\
\hline $55 \mathrm{C}$ & $-0,1581$ \\
\hline $56 \mathrm{C}$ & $-0,1612$ \\
\hline
\end{tabular}

Estos resultados, calculada la ecuación lineal, Cuyo resultado resulta una curva polinómica se refleja en la figura 7. 


\section{Conciencia}

Figura 7

Determinación del tipo de curva y representación de la ecuación

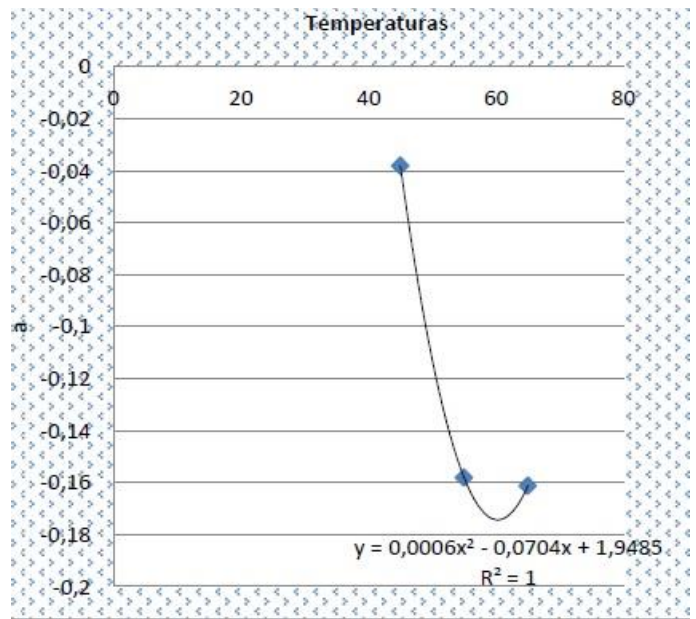

Ya, para la determinación de la variable B en la ortiga, los resultados logrados en el experimento arrojan los siguientes resultados:

Tabla 7

Determinación de constante b para la ecuación del modelo de secado

\begin{tabular}{cc}
\hline Temperaturas & Valores obtenidos \\
\hline $45 \mathrm{C}$ & $-0,3354$ \\
\hline $55 \mathrm{C}$ & $-0,3827$ \\
\hline $56 \mathrm{C}$ & $-0,4751$ \\
\hline
\end{tabular}

Estos resultados, calculada la ecuación lineal, Cuyo resultado resulta una curva polinómica se refleja en la figura 8 .

Figura 8

Determinación del tipo de curva y representación de la ecuación

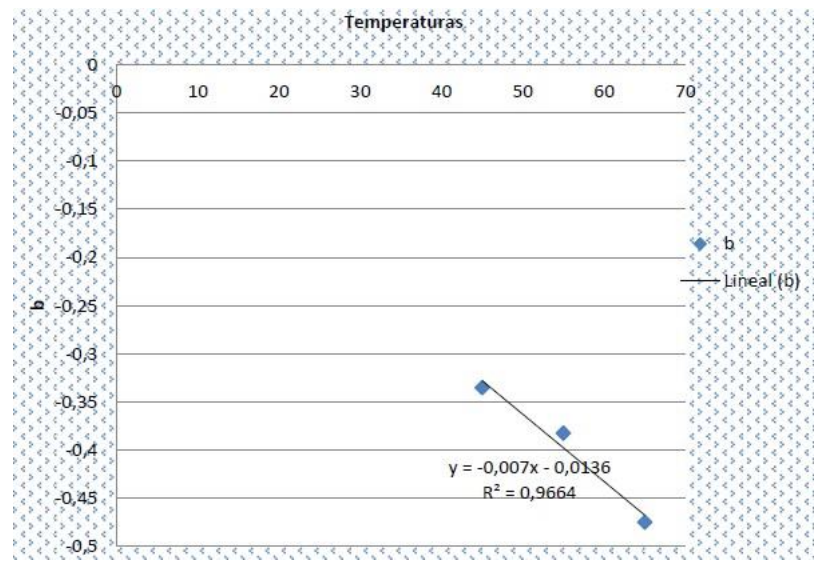


En la determinación de la ratio de secado de matico y ortiga se utilizó el modelo matemático de la curva característica basado en la ecuación $\mathrm{y}=\mathrm{Ax}+\mathrm{B}$; obteniendo valores para la ortiga de $A=-0,0006 x 2-0,0704 x+1.9485$ y $B=-0,007 x-0,0136$; para el caso del matico la ecuación $A=-0,0007 x 2-0,0793 x+2,25$ y $B=-0,007 x-0,0184$ en todas las ecuaciones representa la temperatura de secado.

Estas ecuaciones resueltas desde la estadística inferencial a mediante la herramienta del software SPSS-23, se obtuvieron valores a partir de los cuales se formuló la ratio de secado con ecuación $\mathrm{RS}=\ln (\mathrm{MR})-\mathrm{A} / \mathrm{B}$; siendo $\mathrm{MR}$ la masa relativa de las plantas secas que se determina en un contenido de humedad del $12 \%$ al $14 \%$.

\section{Discusión}

La presente investigación determinó un modelo de ratio de secado en matico y ortiga para los productores locales de la provincia de Chimborazo, los resultados obtenidos demostraron, que, en estas especies de plantas aromáticas, la velocidad del secado es acelerada al igual que el tiempo que implica esta operación. El contenido de humedad de las plantas aromáticas de la investigación llegó a un porcentaje entre el 13\% al 15\%.

Se realizó un secado de matico y ortiga a temperaturas de 45, 55, 65 grados centígrados, hasta conseguir pesos constantes. El tiempo utilizado en el secado de ambas especies de plantas varía de cuatro a seis horas. La ratio de secado abarca el peso de muestreo en cada fase del secado, en función del tiempo, temperatura y humedad dentro del proceso.

En la ratio de secado las curvas de la masa relativa, -MR-, denotan una tendencia al gráfico de una parábola, en tanto que las curvas de secado del antilogaritmo de la masa relativa, LN MR-, denotan una tendencia a una recta. La ratio de secado determinó que a temperaturas de $45^{\circ} \mathrm{C}$ el matico y la ortiga se secan en un periodo de 4 horas, a $55^{\circ} \mathrm{C}$ en 5 horas y a $65^{\circ} \mathrm{C}$ en 6 horas, estos valores coinciden con los obtenidos en la parte experimental. La ratio de secado es utilizada para estandarizar el secado en plantas aromáticas, de manera que puedan ser utilizadas a diferentes condiciones de humedad relativa, velocidad del aire y temperatura.

\section{Conclusiones}

Sobre la base de los objetivos propuestos en esta investigación, se concluye que:

- Es provisto del modelo para la ratio óptima de secado de las plantas de matico y ortiga, a ser comercializados en los mercados serranos. Se logra aplicar la ciencia agroindustrial a resolver problemas económico-sociales y se recomiendan las temperaturas entre 40 y $60 \mathrm{C}$, puesto que son condiciones a las cuáles no se producen cambios en las características fisicoquímicas de estas plantas. 
- Se demuestra que es posible calcular la ratio óptima de secado para elevar la calidad de las plantas previo al proceso de comercialización de éstas. Es necesario realizar la toma de datos del secado en intervalos de tiempo no mayores a una hora, puesto que en esta etapa las plantas pierden la mayor cantidad de agua.

- Son adoptadas prácticas del estado del arte actual en función de perfeccionar el proceso de secado. Énfasis en el uso de los equipos que permitan mantener el contenido de la humedad durante el control de pesos, además de considerar la relación del flujo de aire dentro del secador, ya que, a mayor velocidad de aire caliente, aumenta la pérdida de humedad.

- Al determinar el porcentaje de humedad, es recomendable realizarlo mediante métodos gravimétricos y el uso de una termobalanza con el fin de comprobar los datos logrados.

- Es sugerido generalizar este modelo de secado al resto de Pymes y asociaciones encargadas de esta industria.

\section{Referencias bibliográficas}

Arias F. G. (2006). El proyecto de la Investigación. Introducción a la metodología científica. Caracas. Editorial Episteme. Recuperado desde: https://universoabierto.org/2017/05/22/el-proyecto-de-investigacion-introducciona-la-metodologia-cientifica/

Cabezas, M. (2008). Evaluación nutritiva y nutraceútica de la mora de castilla (Rubus glaucus) deshidratada a tres temperaturas por el método de secado de bandejas. Tesis titulación grado de Ingeniero Agroindustrial. Universidad nacional de Chimborazo. Recuperado desde: http://dspace.espoch.edu.ec/handle/123456789/200

Castellón, J \& Espinoza, W. (2009). Validación de un secador solar de café en pergamino, en fincas de pequeños productores del municipio de San Rafael del Norte. Universidad Nacional de Ingeniería. Nicaragua. Recuperado desde: http://ribuni.uni.edu.ni/1425/

Contreras, C. (2006). Influencia del método de secado en parámetros de calidad relacionados con la estructura y el color de manzana y fresa deshidratadas. Universidad Técnica de Valencia. España. Recuperado desde: https://riunet.upv.es/handle/10251/1932?show=full\&locale-attribute=en 
Cova, W.; Marinelli, H. (2011). Desecación de Productos Agrarios. Universidad tecnológica nacional. Argentina. Recuperado desde: http://www.edutecne.utn.edu.ar/monografias/desecacion_productos_agrarios.pdf

Guerrero Luzuriaga, S. A. (2015). Determinación de un modelo de ratio de secado para el matico y ortiga para los productores locales de la provincia de Chimborazo. Trabajo de grado previo a la obtención del título de Ingeniero Agroindustrial. Universidad nacional del Chimborazo. Recuperado desde: http://dspace.unach.edu.ec/handle/51000/432

Hernández S.; Fernández, A. \& Baptista N. (2006). Metodología de la Investigación. Quinta Edición. Mc GRAW-HILL/INTERAMERICANA DE EDITORES. S.A. DE C.V. Recuperado desde: https://www.esup.edu.pe/descargas/dep_investigacion/Metodologia\%20de\%20la\% 20investigaci\%C3\%B3n\%205ta\%20Edici\%C3\%B3n.pdf

Hernández, E. (2011). Modelado Matemático del secado de madera subtropical Por Convección de Aire Caliente. 2011. Oaxaca. México. Recuperado desde: https://repositoriodigital.ipn.mx/bitstream/123456789/15621/1/Tesis\%20 CIIDIR\%20IPN\%20Oaxaca\%202011.pdf.

Hurtado, I. Toro, G. (2007). Paradigmas y métodos de investigación en tiempos de cambio. Episteme. (5ta Edición) Valencia. Recuperado desde: https://es.scribd.com/document/371098034/Hurtado-y-Toro-paradigmas-yMetodos-de-Investigacion-en-Tiempos-de-Cambio

Jaramillo, A; Narváez, E. (2012). Influencia del método de secado en parámetros de calidad relacionados con la estructura y el color de manzana y fresa deshidratadas. 2012. Universidad técnica de Valencia. Recuperado desde: https://riunet.upv.es/handle/10251/1932?show=full\&locale-attribute=en

Quiroz, R. (2013). Evaluación de la actividad cicatrizante de un gel elaborado a base de los extractos de nogal (Juglans neotrópica diels), ortiga (Urtica dioica 1.), sábila (Aloe vera), en ratones (Mus musculus). Universidad nacional de Chimborazo. Recuperado desde: http://dspace.espoch.edu.ec/handle/123456789/2568

Rojas, M. (2014). Determinación de un modelo de ratio de secado en manzana y manzanilla para lograr la eficiencia de secado del producto en la Provincia de Chimborazo. Universidad nacional de Chimborazo. En línea: http://dspace.unach.edu.ec/bitstream/51000/653/1/UNACH-EC-IET-20140006.pdf 
Roldan G., J. L. (2000). Cómo elaborar un proyecto de investigación. Universidad de Valladolid. España. Recuperado desde: https://alojamientos.uva.es/guia_docente/uploads/2013/429/52504/1/Documento.pd $\underline{\mathrm{f}}$

Shimabukuro, D \& Torres, E. (1992). Estudio técnico de la extracción de aceite esencial de Piper Aduncum L y diseño de planta piloto. Perú. Recuperado desde: http://cybertesis.uni.edu.pe/bitstream/uni/1019/1/shimabukuro_yd.pdf.

Tabar, J. (2011). Obtención de curvas de secado de tomillo. Universidad de Navarra. Recuperado desde: https://academicae.unavarra.es/bitstream/handle/2454/3848/577485.pdf?sequence=1\&isAllowe $\underline{\mathrm{d}=\mathrm{y}}$

Torrecillas, J. (2000). Secado del orujo en lecho fluidizado. Universidad Complutense. España. Recuperado desde: https://eprints.ucm.es/5323/

Varela, J. (2011). Fraccionamiento bioguiado del extracto hidro- etanólico de Aristeguietia glutinosa Lam y elucidación estructural de los principios activos anti - Trypanosoma cruzi. Tesis de grado. Universidad de la República. Uruguay. Recuperado desde: https://www.colibri.udelar.edu.uy/jspui/handle/20.500.12008/1363?mode=full

\section{【 Ciencia}


El artículo que se publica es de exclusiva responsabilidad de los autores y no necesariamente reflejan el pensamiento de la Revista Conciencia Digital.

\section{Liencia}

El artículo queda en propiedad de la revista y, por tanto, su publicación parcial y/o total en otro medio tiene que ser autorizado por el director de la Revista Conciencia Digital.
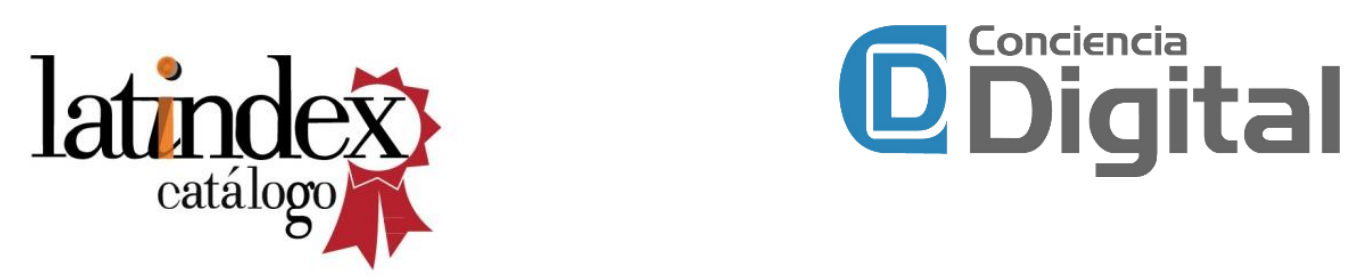

Indexaciones

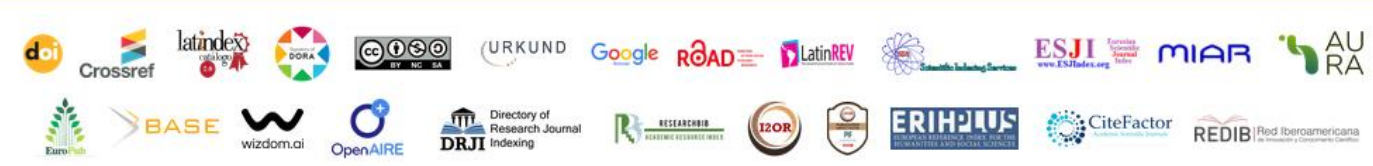

